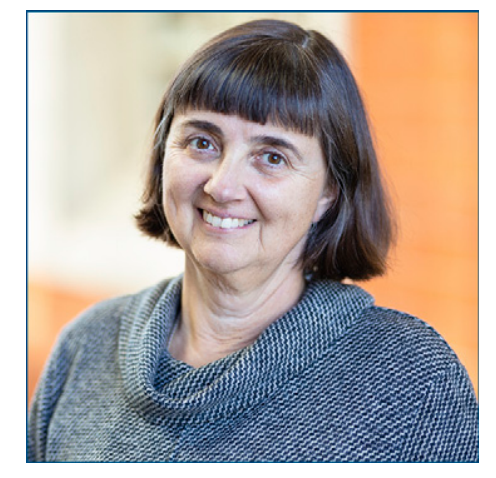

LAURIE ELIT, MD, MSc, $\operatorname{FRCS}(\mathbf{C})$

Laurie Elit, MD, MSc, $\mathrm{FRCS}(\mathrm{C})$, is a

Professor in the Department of Obstetrics and Gynecology and Oncology at McMaster University. She is the division head of Gynecologic Oncology at the Juravinski Hospital and Cancer Centre in Hamilton, Ontario. She leads the Program for Evidence Based Medicine in the Gynecology disease site for Cancer Ontario. doi: $10.6004 /$ jnccn.2020.7556

The ideas and viewpoints expressed in this commentary are those of the authors and do not necessarily represent any policy, position, or program of NCCN.

\section{Routine Imaging or No Routine Imaging, Is That the Question?}

\author{
Laurie Elit, MD, MSc, FRCS(C); Gregory R. Pond, PhD, PStat; and \\ Mark N. Levine, CM, MD, MSc, FRCP(C)
}

$\mathbf{P}$ atients with cancer who have completed their primary, and potentially curative, therapy often ask their physician to perform follow-up cancer testing. These patients are seeking reassurance that they remain cancer-free while also looking to detect potential early recurrence of the cancer. The underlying premise is that detecting recurrence at an early stage can lead to earlier interventions that will eradicate the cancer or prolong survival. However, diagnostic testing is only beneficial if interventions exist that can alter the clinical course of the disease. Otherwise, the only impact of early detection is to give the patient more time with the disease (lead-time bias). Introducing potentially toxic treatments such as chemotherapy to asymptomatic patients can have a profound detrimental impact on their quality of life, despite not prolonging survival. Additionally, increased use of surveillance testing can amplify patient anxiety from heightened awareness of upcoming investigations and as they wait for results. Table 1 outlines some potential benefits and harms related to increased surveillance.

In the context of patients with advanced epithelial ovarian cancer, in a randomized trial of surveillance with and without CA-125 monitoring, Rustin et al ${ }^{1}$ clearly showed that earlier identification of recurrent disease resulted in more lines of systemic therapy but did not lead to longer survival in patients from Europe. Recently, a randomized trial on secondary surgical cytoreduction from the Gynecologic Oncology Group ${ }^{2}$ again showed no benefit, which suggests that early detection of tumor recurrence would not affect survival. Thus, there appears to be strong evidence against the use of more intensive follow-up imaging in this patient population. Based on these considerations, the Society of Gynecologic Oncology recommended against routine posttreatment surveillance imaging. ${ }^{3,4}$

The article by Green et $\mathrm{al}^{5}{ }^{5}$ elsewhere in this issue, further examines this question. As a result of hypothesized differences that might exist within the United States population, Green et al ${ }^{5}$ conducted a retrospective study on patients with stage II-IV epithelial ovarian cancer treated at one institution in 2001 through 2017. They explored whether there was a relationship between the number of imaging tests performed within the first year after completion of primary chemotherapy and time to recurrence and overall survival. ${ }^{5}$ Patients were included if they had at least 1 year of follow-up. Physicians were divided into 2 groups: those with frequent imaging practices (>1 scan every 12 months) and those with less frequent imaging practices. Median time to recurrence was shorter for patients treated by physicians who used frequent imaging versus less frequent imaging (18.0 vs 19.2 months; hazard ratio [HR], 1.33); however, this did not translate into any difference in overall survival (HR, 1.10). In summary, these results further support the argument that increased surveillance does not result in increased survival, while also emphasizing that it might in fact cause increased harm, due to increased lead-time bias.

For clinicians who need to interpret study results and apply them to their individual practice, the quality of evidence is an important consideration. How does one evaluate the quality of studies, and how much weight should one put on the

See page $\mathbf{4 1 4}$ for related article. 


\section{Table 1. Potential Benefits Versus Harms of Surveillance Testing}

\begin{tabular}{|ll|}
\hline Benefits & Harms \\
\hline Enhanced overall survival & Increased duration and severity of anxiety \\
\hline Enhanced progression-free survival & Additional investigations and procedure \\
\hline Reduced time to recurrence & Possible false-positive investigations \\
\hline Enhanced time without symptoms & Increased time on treatment \\
\hline & Increased treatment-related adverse events \\
\hline & Increased costs to patients \\
\hline
\end{tabular}

evidence provided by this observational study reported in this issue of JNCCN? ${ }^{5}$ Clinicians can properly evaluate the quality of cohort studies such as this one by applying previously published criteria. ${ }^{6-8}$

First, was there a clear inception cohort of patients who were assembled and followed in a uniform manner?

In this case, the answer is no. The investigators started with approximately 1,800 patients in their cohort but ended up with 543 patients and 23 providers in one group and 141 patients and 4 providers in the other group. More than 50\% of patients were lost to follow-up and the remaining patients were highly selected. Table 1 in the manuscript ${ }^{5}$ suggests that the 2 groups were balanced for baseline characteristics; however, it is unclear how different this cohort of patients is from those who were excluded, and how the 2 groups compared over time. This study was performed over a 16-year period, when treatments and providers probably would have changed. Further, no rationale is provided for defining high versus low surveillance based on a cutoff of $>1$ versus $\leq 1$ scan per year. As a result, it is not clear whether the results would change if differing points were used.

Second, was there complete follow-up of all patients in the cohorts? We found a lack of clarity on the number of patients lost to follow-up in the 2 cohorts. Particularly, patients who were enrolled onto clinical trials, lost to follow-up, or who died with no prior recurrence were excluded from this analysis. Certainly, the individual provider could have a large influence on clinical trial enrollment and probably an effect on whether patients choose to return to the treatment center for follow-up. These exclusions could result in unmeasured bias in the reported results.

Third, was there adjustment for potential confounders? In this study, no statistical adjustments were made in the form of regression models, and providers may have changed surveillance patterns based on characteristics such as disease stage, baseline CA-125 level, or patient age.

Finally, how generalizable are the results? As a retrospective cohort from a single, high-volume tertiary care institution, these results may not be generalizable to many practitioners at other institutions who may have different practice patterns, particularly given that the low-imaging-frequency cohort is based on a group of only 4 providers. Further, during the 16-year period of this study, practice patterns would likely have changed substantially due to the introduction of PARP inhibitors or bevacizumab. The study included no data on whether patients receiving maintenance bevacizumab or PARP inhibitors were excluded from analysis or, if included, how their surveillance patterns might have changed based on receiving these treatments.

In summary, the study by Green et $\mathrm{a}^{5}$ explored the question, "Does more intensive radiologic imaging provide superior survival to regular care?" The findings did not show any benefit for more frequent imaging in the surveillance population. Based on the available evidence, we recommend following the Society of

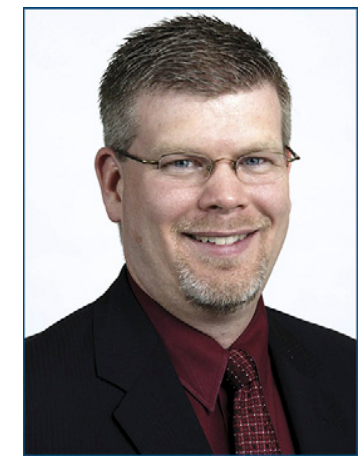

GREGORY R. POND, PhD, PStat

Gregory R. Pond, PhD, PStat, is an Associate Professor in the Departments of Oncology, and Health Research Methods, Evidence, and Impact (HEI), and a Research Scientist in the Escarpment Cancer Research Institute at McMaster University, Hamilton, Ontario, Canada. Dr. Pond is also a Senior Investigator at the Ontario Institute for Cancer Research. 


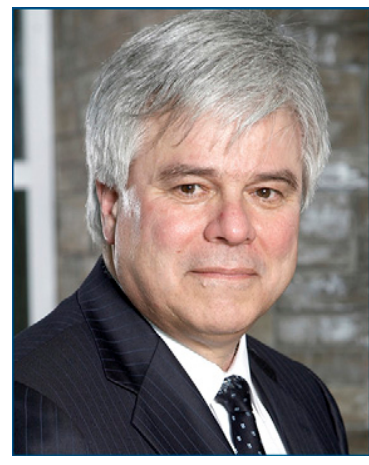

MARK N. LEVINE, CM, MD, $\operatorname{MSc}, \operatorname{FRCP}(C)$

Mark N. Levine, CM, MD, MSc, $\operatorname{FRCP}(C)$, is a Professor in the Department of Oncology at McMaster University, Hamilton, Ontario, Canada. He holds the Buffett Taylor Chair in Breast Cancer Research at McMaster University. He has practiced medical oncology for 36 years. Dr. Levine has been an active researcher in clinical trials and health services research in the areas of breast cancer and venous thromboembolism. A number of the trials he has conducted have been landmark studies influencing health care in both Canada and internationally. Dr. Levine also has over 310 publications in peer-reviewed journals and has brought significant research funding to McMaster University. Dr. Levine has held numerous prominent professional positions throughout his career. In addition, Dr. Levine has been the recipient of several prestigious accolades that include the O. Harold Warwick Prize from the National Cancer Institute of Canada and the Order of Canada.
Gynecologic Oncology guidelines against routine posttreatment surveillance imaging. Additionally, in contrast to the authors, we believe that a randomized trial evaluating novel investigations or strategies for surveillance is still possible. However, before such a trial is conducted, researchers should be sure there is an intervention that will prolong the survival of patients with recurrent ovarian cancer that presents with a small disease burden.

Disclosures: The authors have disclosed that they have no financial interests, arrangements, or affiliations with the manufacturers of any products discussed in this article or their competitors.

Correspondence: Laurie Elit, MD, MSc, FRCS(C), Juravinski Cancer Centre, 699 Concession Street, Hamilton, Ontario, Canada. Email: elitlor@hhsc.ca

\section{References}

1. Rustin GJ, van der Burg ME, Griffin CL et al. Early versus delayed treatment of relapsed ovarian cancer (MRC OV05/EORTC 55955): a randomized trial. Lancet 2010;376:1155-1163.

2. Coleman RL, Spirtos NM, Enserro D, et al. Secondary surgical cytoreduction for recurrent ovarian cancer. N Engl J Med 2019;318:1929-1939.

3. Salani R, Khanna N, Frimer M, et al. An update on post-treatment surveillance and diagnosis of recurrence in women with gynecologic malignancy: Society of Gynecologic Oncology (SGO) recommendations. Gynecol Oncol 2017;146:3-10.

4. Rimel BJ, Burke WM, Higgins RV et al. Improving quality and decreasing cost in gynecologic oncology care: Society of Gynecologic Oncology recommendations for clinical practice. Gynecol Oncol 2015;137:280-284.

5. Green AK, Korenstein D, Aghajanian C, et al, Impact of provider imaging practices on survival outcomes in advanced ovarian cancer. J Natl Compr Canc Netw 2020;18:414-419.

6. Sackett D, Guyatt G, Tugwell P, Haynes RB. Clinical Epidemiology: How to Do Clinical Practice Research. Philadelphia, PA: JB Lipincott Williams and Wilkins; 2005.

7. Laupacis A, Wells, G, Richardson WS, Tugwell P. Users' guides to the medical literature. V. How to use an article about prognosis. Evidence-Based Medicine Working Group. JAMA 1994;272:234-237.

8. Levine MN, Julian J. Registries that show efficacy: good, but not good enough. J Clin Oncol 2008;26: 5316-5319. 